This is a self-archived version of an original article. This version may differ from the original in pagination and typographic details.

Author(s): Nandi, Debanjan; Rajala, Tapio; Schultz, Timo

Title: A Density Result for Homogeneous Sobolev Spaces on Planar Domains

Year: 2019

Version: Accepted version (Final draft)

Copyright: (C) Springer Nature B.V. 2018

Rights: In Copyright

Rights url: http://rightsstatements.org/page//nC/1.0/?language=en

Please cite the original version:

Nandi, D., Rajala, T., \& Schultz, T. (2019). A Density Result for Homogeneous Sobolev Spaces on Planar Domains. Potential Analysis, 51(4), 483-498. https://doi.org/10.1007/s11118-018-9720-8 


\title{
A DENSITY RESULT FOR HOMOGENEOUS SOBOLEV SPACES ON PLANAR DOMAINS
}

\author{
DEBANJAN NANDI, TAPIO RAJALA, AND TIMO SCHULTZ
}

\begin{abstract}
We show that in a bounded simply connected planar domain $\Omega$ the smooth Sobolev functions $W^{k, \infty}(\Omega) \cap C^{\infty}(\Omega)$ are dense in the homogeneous Sobolev spaces $L^{k, p}(\Omega)$.
\end{abstract}

\section{INTRODUCTION}

By the result of Meyers-Serrin [16] it is known that $C^{\infty}(\Omega)$ is dense in $W^{k, p}(\Omega)$ for every open set $\Omega$ in $\mathbb{R}^{d}$. The space $C^{\infty}\left(\mathbb{R}^{d}\right)$ is not always dense in $W^{k, p}(\Omega)$, for example when $\Omega$ is a slit disk. However, a slit disk is not a very appealing example as it is not the interior of its closure. Counterexamples for the density satisfying $\Omega=\operatorname{int}(\bar{\Omega})$ were given by Amick [1] and Kolsrud [9]. In fact, in these examples even $C(\bar{\Omega})$ is not dense in $W^{k, p}(\Omega)$. Going further in counterexamples, O'Farrell [18] constructed a domain satisfying $\Omega=\operatorname{int}(\bar{\Omega})$ where $W^{k, \infty}(\Omega)$ is not dense in $W^{k, p}(\Omega)$ for any $k$ and $p$. The domain constructed by O'Farrell was infinitely connected. From the recent results of Koskela-Zhang [14] and Koskela-Rajala-Zhang [13] we can conclude that this is necessary for such constructions in the plane, since $W^{1, \infty}(\Omega)$ is dense in $W^{1, p}(\Omega)$ for all finitely connected bounded planar domains (see also the earlier work by Giacomini-Trebeschi [4]). Further examples of domains where $W^{1, p}(\Omega)$ is not dense in $W^{1, q}(\Omega)$ were constructed by Koskela [11] and Koskela-Rajala-Zhang [13].

In this note we continue the study of density of $W^{k, \infty}(\Omega)$ in $W^{k, p}(\Omega)$. Let us remark that such density clearly holds in the case where the Sobolev functions in $W^{k, p}(\Omega)$ can be extended to Sobolev functions defined on the whole $\mathbb{R}^{2}$. By work of Jones [8], this is true when $\partial \Omega$ is a quasi-circle. (See also the works [6, 5, 7].) Geometric characterizations of Sobolev extension domains are known, especially in the planar simply connected domains when $k=1$, see [3, 10, 19, 12].

Being an extension domain is only a sufficient condition for the density. For example, there are Jordan domains $\Omega$ and functions $f \in W^{1, p}(\Omega)$ that cannot be extended to a function in $W^{1, p}\left(\mathbb{R}^{2}\right)$. However, global smooth functions are dense in $W^{1, p}(\Omega)$ for any Jordan domain and any $p \in[1, \infty]$, see Lewis [15] and Koskela-Zhang [14]. For $W^{k, p}(\Omega)$ with $k \geq 2$ this is still unknown.

In 20. Smith-Stanoyevitch-Stegenga studied the density of $C^{\infty}\left(\mathbb{R}^{2}\right)$ as well as the density of functions in $C^{\infty}(\Omega)$ with bounded derivatives, in $W^{k, p}(\Omega)$. For the latter class they

Date: March 10, 2019.

2000 Mathematics Subject Classification. Primary 46E35.

Key words and phrases. Sobolev space, homogeneous Sobolev space, density.

All authors partially supported by the Academy of Finland. 
obtained a density result assuming $\Omega$ to be starshaped or to satisfy an interior segment condition. For the smaller class of functions $C^{\infty}\left(\mathbb{R}^{2}\right)$ they also required an extra assumption on the boundary points to be $m_{2}$-limit points. (See also Bishop [2] for a counterexample on a related question.)

The result of Koskela-Zhang 14 showing that $W^{1, \infty}(\Omega)$ is dense in $W^{1, p}(\Omega)$ for every bounded simply connected planar domain was generalized to higher dimensions by KoskelaRajala-Zhang [13]. They showed that simply connectedness is not sufficient to give such a density result, but Gromov hyperbolicity in the hyperbolic distance is. In this paper we provide another generalization to the Koskela-Zhang result by going to higher order Sobolev spaces. We show that if we restrict attention to the homogenous norm, then being simply connected is sufficient for domains in the plane.

For a domain $\Omega \subset \mathbb{R}^{2}$ and $p \in[1, \infty)$, by homogenous Sobolev space $L^{k, p}(\Omega)$ we mean functions with $p$-integrable distributional derivatives of order $k$;

$$
L^{k, p}(\Omega)=\left\{u \in L_{l o c}^{1}(\Omega): \nabla^{\alpha} u \in L^{p}(\Omega), \text { if }|\alpha|=k\right\},
$$

with semi-norm $\sum_{|\alpha|=k}\left\|\nabla^{\alpha} u\right\|_{L^{p}(\Omega)}$, where $\alpha$ is any 2-vector of non-negative integers and $|\alpha|$ is its $\ell_{1}$-norm. The (non-homogenous) Sobolev space $W^{k, p}(\Omega)$ is defined as

$$
W^{k, p}(\Omega)=\left\{u \in L_{l o c}^{1}(\Omega): \nabla^{\alpha} u \in L^{p}(\Omega) \text {, if }|\alpha| \leq k\right\},
$$

with norm $\sum_{|\alpha| \leq k}\left\|\nabla^{\alpha} u\right\|_{L^{p}(\Omega)}$.

Theorem 1.1. Let $k \in \mathbb{N}, p \in[1, \infty)$ and $\Omega \subset \mathbb{R}^{2}$ be a bounded simply connected domain. Then the subspace $W^{k, \infty}(\Omega) \cap C^{\infty}(\Omega)$ is dense in the space $L^{k, p}(\Omega)$.

The approach in [13] differs from ours in that there the approximating functions are defined via shifting matters to the disk via the Riemann mapping. Instead, we directly make a Whitney decomposition of the domain and a rough reflection to define our approximating sequence. We achieve this via an elementary use of simply connectedness in the plane. In both of these approaches the values of the function in a suitable compact set are used to define a smooth function in the entire domain which approximates the original function in Sobolev norm. For this we employ similar tools as used by Jones in [8].

In $p$-Poincaré domains, that is domains $\Omega$ where a $p$-Poincaré inequality

$$
\int_{\Omega}\left|u-u_{D}\right|^{p} \mathrm{~d} x \leq C \int_{\Omega}|\nabla u|^{p} \mathrm{~d} x
$$

holds, we can bound the integrals of the lower order derivatives by the integrals of the higher order ones and thus we obtain the following corollary to our Theorem 1.1 .

Corollary 1.2. Let $k \in \mathbb{N}, p \in[1, \infty)$ and $\Omega \subset \mathbb{R}^{2}$ be a bounded simply connected $p$ Poincaré domain. Then $W^{k, \infty}(\Omega) \cap C^{\infty}(\Omega)$ is dense in the space $W^{k, p}(\Omega)$.

For instance Hölder-domains are $p$-Poincaré domains for $p \geq 2$, see Smith-Stegenga [21]. It still remains an open question whether Corollary 1.2 holds if one drops the assumption of being a $p$-Poincaré domain.

Next we come to the question of density of $C^{\infty}\left(\mathbb{R}^{2}\right)$ functions in $L^{k, p}(\Omega)$ in our setting of bounded simply connected domains. We have the following corollary which is analogous to 
[14, Corollary 1.2], where it is shown that $\Omega$ being Jordan is sufficient. A small modification of the argument there applies to our situation as well. See the end of Section 4 for the proof.

Corollary 1.3. Let $k \in \mathbb{N}, p \in[1, \infty)$ and $\Omega \subset \mathbb{R}^{2}$ be a Jordan domain. Then $C^{\infty}\left(\mathbb{R}^{2}\right)$ is dense in the space $L^{k, p}(\Omega)$.

In Section 2, we collect the necessary ingredients which will be used for defining the approximating sequence; these include a suitable Whitney-type decomposition of a simply connected domain and a local polynomial approximation of Sobolev functions. In Section 3 we describe a partition of the domain using the Whitney-type decomposition of Section 2. which is needed for obtaining a suitable partition of unity. Then in Section 4, we define the approximating sequence and present the necessary estimates for proving Theorem 1.1 and Corollary 1.3 .

\section{PRELiminaRies}

For sets $A, B \subset \mathbb{R}^{2}$ we denote the diameter of $A$ by $\operatorname{diam}(A)$ and the distance between $A$ and $B$ by $\operatorname{dist}(A, B)$. We denote by $B(x, r)$ the open ball with center $x \in \mathbb{R}^{2}$ and radius $r>0$ and more generally, by $B(A, r)$ the open $r$-neighbourhood of a set $A \subset \mathbb{R}^{2}$. Given a connected set $E \subset \mathbb{R}^{2}$ and points $x, y \in E$, we define the inner distance $\mathrm{d}_{E}(x, y)$ between $x$ and $y$ in $E$ to be the infimum of lengths of curves in $E$ joining $x$ to $y$. (Notice that in general the infimum might have value $\infty$.) We write the inner distance in $E$ between sets $A, B \subset E$ as $\operatorname{dist}_{E}(A, B)$.

With a slight abuse of notation, by a curve $\gamma$ we refer to both, a continuous mapping $\gamma:[0,1] \rightarrow \mathbb{R}^{2}$ and its image $\gamma([0,1])$. Given two curves $\gamma_{1}, \gamma_{2}:[0,1] \rightarrow \mathbb{R}^{2}$ such that $\gamma_{1}(1)=\gamma_{2}(0)$, we denote by $\gamma_{1} * \gamma_{2}:[0,1] \rightarrow \mathbb{R}^{2}$ the concatenated curve $\gamma_{1} * \gamma_{2}(t)=\gamma_{1}(2 t)$ for $t \leq 1 / 2$ and $\gamma_{1} * \gamma_{2}(t)=2 t-1$ for $t \geq 1 / 2$. We denote the length of a curve $\gamma$ by $L(\gamma)$.

We will use the following facts in plane topology whose proofs can be found in the book of Newman [17, Chapter VI, Theorem 5.1 and Chapter V, Theorem 11.8].

Lemma 2.1. Let $\Omega$ be a simply connected domain in $\mathbb{R}^{2}$ and $\gamma:[0,1] \rightarrow \mathbb{R}^{2}$ a continuous curve that is injective on $(0,1)$, whose endpoints $\gamma(0)$ and $\gamma(1)$ are in $\partial \Omega$ and interior $\gamma((0,1))$ in $\Omega$. Then $\Omega \backslash \gamma$ has two connected components, both of which are simply connected.

In the case where $\Omega$ is Jordan and $\gamma$ is homeomorphic to a closed interval, the two connected components of $\Omega \backslash \gamma$ have boundaries $\gamma \cup J_{1}$ and $\gamma \cup J_{2}$, where $J_{1}$ and $J_{2}$ are the two connected components of $\partial \Omega \backslash \gamma$.

2.1. A dyadic decomposition. Although it is standard to consider a Whitney decomposition of a domain in $\mathbb{R}^{d}$ (see for instance Whitney [23] or the book of Stein [22, Chapter $\mathrm{VI}]$ ), we will use a precise construction of such a decomposition. We present this construction below. Here and later on we denote the sidelength of a square $Q$ by $l(Q)$.

For notational convenience we start the Whitney decomposition below from squares with sidelength $2^{-1}$. Formally, by rescaling, we may consider all bounded domains $\Omega \subset \mathbb{R}^{2}$ to 
have $\operatorname{diam}(\Omega) \leq 1$ in which case no Whitney decomposition would have squares larger than the ones used below regardless of the starting scale.

Definition 2.2 (Whitney decomposition). Let $\Omega \subset \mathbb{R}^{2}$ be a bounded (simply connected) open set. Let $\mathcal{Q}_{n}$ be the collection of all closed dyadic squares of sidelength $2^{-n}$. Define a Whitney decomposition as $\tilde{\mathcal{F}}:=\bigcup_{n \in \mathbb{N}} \tilde{\mathcal{F}}_{n}$ where the sets $\tilde{\mathcal{F}}_{n}$ are defined recursively as follows. Define

$$
\tilde{\mathcal{F}}_{1}:=\left\{Q \in \mathcal{Q}_{1}: \bigcup_{\substack{Q^{\prime} \in \mathcal{Q}_{1} \\ Q^{\prime} \cap Q \neq \emptyset}} Q^{\prime} \subset \Omega\right\}
$$

and

$$
\tilde{\mathcal{F}}_{n+1}:=\left\{Q \in \mathcal{Q}_{n+1}: Q \not \subset \tilde{F}_{n} \text { and } \bigcup_{\substack{Q^{\prime} \in \mathcal{Q}_{n+1} \\ Q^{\prime} \cap Q \neq \emptyset}} Q^{\prime} \subset \Omega\right\},
$$

where $\tilde{F}_{n}=\bigcup_{j \leq n} \bigcup_{Q \in \tilde{\mathcal{F}}_{j}} Q$.

Lemma 2.3. A Whitney decomposition given by Definition 2.2 has the following properties.

(W1) $\Omega=\bigcup_{Q \in \tilde{\mathcal{F}}} Q$

(W2) $l(Q)<\operatorname{dist}\left(Q, \Omega^{c}\right) \leq 3 \sqrt{2} l(Q)=3 \operatorname{diam}(Q)$ for all $Q \in \tilde{\mathcal{F}}$

(W3) int $Q_{1} \cap \operatorname{int} Q_{2}=\emptyset$ for all $Q_{1}, Q_{2} \in \tilde{\mathcal{F}}, Q_{1} \neq Q_{2}$

(W4) If $Q_{1}, Q_{2} \in \tilde{\mathcal{F}}$ and $Q_{1} \cap Q_{2} \neq \emptyset$, then $\frac{l\left(Q_{1}\right)}{l\left(Q_{2}\right)} \leq 2$.

Proof. Although the proof is very elementary, we give it here for completeness.

For (W1), take any $x \in \Omega$ and $n \in \mathbb{N}$ such that $x \in Q \in \mathcal{Q}_{n}$, where $2^{-n+2} \sqrt{2}<$ $\operatorname{dist}\left(x, \Omega^{c}\right) \leq 2^{-n+3} \sqrt{2}$. Then for any $Q^{\prime} \in \mathcal{Q}_{n}$ with $Q^{\prime} \cap Q \neq \emptyset$ we have $Q^{\prime} \subset \Omega$. Hence by definition either $Q \in \tilde{F}_{n}$ or $x \in Q \subset Q^{\prime \prime} \in \tilde{\mathcal{F}}_{i}$ for some $i<n$.

In order to see (W2), let $Q \in \tilde{\mathcal{F}}_{n}$. Then all $Q^{\prime} \subset \Omega$ for all $Q^{\prime} \in \mathcal{Q}_{n}$ with $Q^{\prime} \cap Q \neq \emptyset$. Consequently, $\operatorname{dist}\left(Q, \Omega^{c}\right)>2^{-n}=l(Q)$. For the upper bound, suppose $\operatorname{dist} Q, \Omega^{c}>$ $3 \sqrt{2} 2^{-n}$. Let $Q_{2} \in \mathcal{Q}_{n-1}$ be such that $Q \subset Q_{2}$. Then $\operatorname{dist}\left(Q_{2}, \Omega^{c}\right)>\sqrt{2} 2^{-n+1}$ and so $Q_{3} \subset \Omega$ for all $Q_{3} \in \mathcal{Q}_{n-1}$ for which $Q_{2} \cap Q_{3} \neq \emptyset$. Thus $Q_{2} \in \tilde{F}_{n-1}$ or $Q_{2} \subset Q_{4} \in \tilde{F}_{i}$ for some $i<n-1$. In either case, $Q \notin \tilde{\mathcal{F}}_{n}$ giving a contradiction.

Property (W3) holds by the recursion in the definition and the fact that the dyadic squares are nested.

Suppose (W4) is not true. Then there exist $Q_{1} \in \tilde{\mathcal{F}}_{n}$ and $Q_{2} \in \tilde{\mathcal{F}}_{m}$ with $n<m-1$ and $Q_{1} \cap Q_{2} \neq \emptyset$. Let $Q_{3} \in \tilde{F}_{n+1}$ be such that $Q_{2} \subset Q_{3}$. Then

$$
\bigcup_{\substack{Q^{\prime} \in \mathcal{Q}_{n+1} \\ Q^{\prime} \cap Q_{3} \neq \emptyset}} Q^{\prime} \subset \bigcup_{\substack{Q^{\prime} \in \mathcal{Q}_{n} \\ Q^{\prime} \cap Q_{1} \neq \emptyset}} Q^{\prime} \subset \Omega
$$

and so either $Q_{3} \in \tilde{\mathcal{F}}_{n+1}$ or $Q_{3} \subset \tilde{F}_{n}$. In both cases $Q_{2} \subset \tilde{F}_{n+1}$ and so $Q_{2} \notin \tilde{\mathcal{F}}_{m}$. 
By a chain of dyadic squares $\left\{Q_{i}\right\}_{i=1}^{m}$ we mean a collection of sets $Q_{i} \in \tilde{\mathcal{F}}$ such that $Q_{i} \cap Q_{i+1}$ is a non-degenerate line segment for all $i \in\{1, \ldots, m-1\}$. We say that the chain connects $Q_{1}$ and $Q_{m}$.

2.2. Approximating polynomials. We record here the following two Lemmas from 8 which will be used when estimating the approximation in Section 4. By $|E|$ we denote the Lebesgue measure of a set $E \subset \mathbb{R}^{2}$.

Lemma 2.4 (Lemma 2.1, [8]). Let $Q$ be any square in $\mathbb{R}^{2}$ and $P$ be a polynomial of degree $k$ defined in $\mathbb{R}^{2}$. Let $E, F \subset Q$ be such that $|E|,|F|>\eta|Q|$ where $\eta>0$. Then

$$
\|P\|_{L^{p}(E)} \leq C(\eta, k)\|P\|_{L^{p}(F)} .
$$

Given a function $u \in C^{\infty}(\Omega)$ and a bounded set $E \subset \Omega$ with $|E|>0$, we define (see [8]) the polynomial approximation of $u$ in $E, P_{k}(u, E)$ to be the polynomial of order $k-1$ which satisfies

$$
\int_{E} \nabla^{\alpha}\left(u-P_{k}(u, E)\right)=0
$$

for each $\alpha=\left(\alpha_{1}, \alpha_{2}\right)$ such that $|\alpha|=\alpha_{1}+\alpha_{2} \leq k-1$. Once $k$ is fixed, we denote the polynomial approximation of $u$ in a dyadic square $Q$ as $P_{Q}$

The next lemma is a consequence of Poincaré inequality for Lipschitz domains.

Lemma 2.5 (Lemma 3.1, [8]). Let $\Omega \subset \mathbb{R}^{2}$ be a bounded simply connected domain and $\tilde{\mathcal{F}}$ a Whitney decomposition of $\Omega$. Fix $\alpha$ such that $|\alpha| \leq k$. Let $\left\{Q_{i}\right\}_{i=1}^{m}$ in $\tilde{\mathcal{F}}$ be a chain of dyadic squares in $\tilde{\mathcal{F}}$. Then we have

$$
\left\|\nabla^{\alpha}\left(P_{Q_{1}}-P_{Q_{m}}\right)\right\|_{L^{p}\left(Q_{1}\right)} \leq C l\left(Q_{1}\right)^{k-|\alpha|}\left\|\nabla^{k} u\right\|_{L^{p}\left(\bigcup_{i=1}^{m} Q_{i}\right)},
$$

where $\nabla^{k} u$ is the vector $\left(\nabla^{\alpha} u\right)_{|\alpha|=k}$ normed by the $\ell_{2}$-norm and $C=C(m)$.

In what follows, given $\beta=\left(\beta_{1}, \beta_{2}\right)$ and $\alpha=\left(\alpha_{1}, \alpha_{2}\right)$, we write $\beta \leq \alpha$ if the inequality holds coordinate-wise.

\section{Decomposition of the Domain}

From now on we fix a bounded simply connected domain $\Omega \subset \mathbb{R}^{2}$ and a Whitney decomposition $\tilde{\mathcal{F}}$ of $\Omega$ given by Definition 2.2. For our purposes we need to choose at each level a nice enough subcollection of $\tilde{\mathcal{F}}_{n}$, namely we take connected components of the Whitney decomposition (see Figure 1). More precisely we fix $Q_{0} \in \tilde{\mathcal{F}}_{1}$ and for each $n \in \mathbb{N}$ let $C_{n}$ be the connected component of the interior of $\tilde{F}_{n}$ that has $\operatorname{int} Q_{0}$ as a subset. We define

$$
\mathcal{F}_{n, j}:=\left\{Q \in \tilde{\mathcal{F}}_{j}: \operatorname{int} Q \subset C_{n}\right\}
$$

and using this the families of squares

$$
\mathcal{F}_{n}:=\mathcal{F}_{n, n}, \quad \mathcal{D}_{n}:=\bigcup_{j \leq n} \mathcal{F}_{n, j}
$$




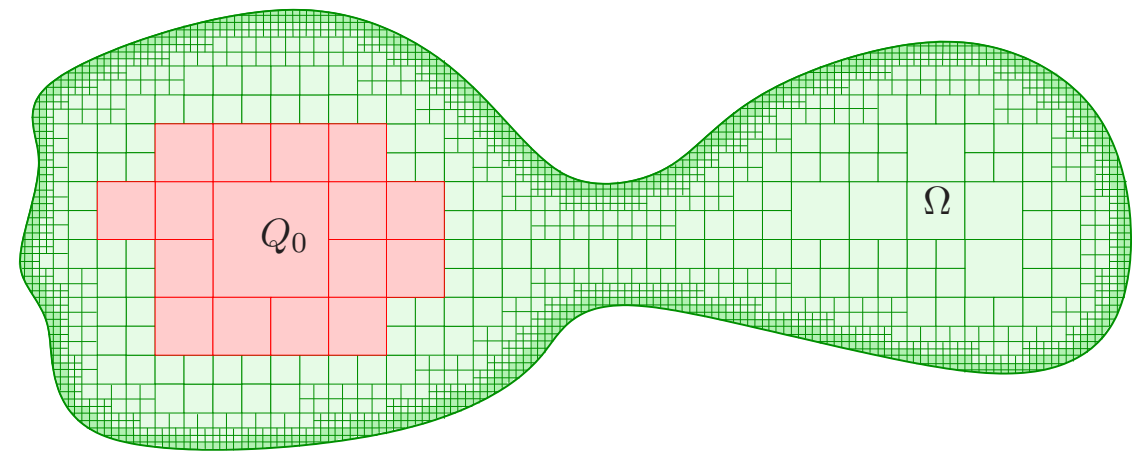

FiguRE 1. A core part $D_{n}$ is selected from the Whitney decomposition of $\Omega$ by taking the connected component containing $Q_{0}$ of the interior of the union of Whitney squares with sidelength at least $2^{-n}$.

and the corresponding sets for two of the above collections by

$$
F_{n}:=\bigcup_{Q \in \mathcal{F}_{n}} Q \text { and } D_{n}:=\bigcup_{Q \in \mathcal{D}_{n}} Q=\bar{C}_{n} .
$$

The collection of boundary layer squares in $\mathcal{D}_{n}$ is denoted by

$$
\partial \mathcal{D}_{n}:=\left\{Q \in \mathcal{D}_{n}: Q \cap \overline{\left(\Omega \backslash D_{n}\right)} \neq \emptyset\right\} .
$$

With this notation we have the following lemma.

Lemma 3.1. The above collections have the properties:

(i) $\mathcal{D}_{n} \subset \mathcal{D}_{n+1}$ for all $k \in \mathbb{N}$.

(ii) $\Omega=\bigcup_{n \in \mathbb{N}} D_{n}$.

(iii) If $Q_{1}, Q_{2} \in \mathcal{F}_{n}$ and $Q_{1} \cap Q_{2}$ is a singleton, then there exists $Q_{3} \in \mathcal{D}_{n}$ for which $Q_{1} \cap Q_{2} \cap Q_{3} \neq \emptyset$.

(iv) If $Q \in \partial \mathcal{D}_{n}$, then $Q \in \mathcal{F}_{n}$.

(v) If $Q \in \partial \mathcal{D}_{n}$, then $Q \cap \overline{\left(\Omega \backslash \tilde{F}_{n}\right)} \neq \emptyset$.

(vi) The set $C_{n}$ is simply connected.

Proof. The property (i) is obvious by the definitions of $\mathcal{F}_{n, j}$ and $\mathcal{D}_{n}$ since $C_{n} \subset C_{n+1}$.

For (ii) it suffices to prove that for every $Q \in \tilde{\mathcal{F}}$ there exists $n \in \mathbb{N}$ so that $Q \in \mathcal{D}_{n}$. Let $Q \in \mathcal{F}_{n}$. Since $\Omega$ is connected and open, there exists a path $\gamma$ in $\Omega$ joining $Q$ to $Q_{0}$. By the fact that $\tilde{F}_{j} \subset \operatorname{int} \tilde{F}_{j+1}$ and the property $(\mathrm{W} 1)$ of the decomposition $\tilde{\mathcal{F}}$ we have that $\Omega=\cup_{j \in \mathbb{N}}$ int $\tilde{F}_{j}$. Then by the compactness of $\gamma$ there exists $m \geq n$ so that $\gamma \subset \operatorname{int} \tilde{F}_{m}$. Hence $Q \in \mathcal{D}_{m}$.

For (iii) let $Q_{1}, Q_{2} \in \mathcal{F}_{n}$ be so that $Q_{1} \cap Q_{2}$ is a singleton $\{q\}$. Assume that the claim is false. Then for the two squares $Q \in \mathcal{Q}_{n}$ that intersect both $Q_{1}$ and $Q_{2}$ it is true that $Q \notin \tilde{\mathcal{F}}_{n}$ and $Q \not \subset Q^{\prime}$ for all $Q^{\prime} \in \tilde{\mathcal{F}}_{n-1}$. Let $q_{1}$ and $q_{2}$ be the centres of the squares $Q_{1}$ and $Q_{2}$ respectively. Consider a curve $\gamma^{\prime}:[0,1] \rightarrow \Omega$ for which $\gamma_{0}^{\prime}=q_{1}, \gamma_{1}^{\prime}=q_{1}$ and $\gamma^{\prime} \subset C_{n}$. Such a curve exists by the definition of $C_{n}$. We may also assume that $\gamma^{\prime}$ is an injective 


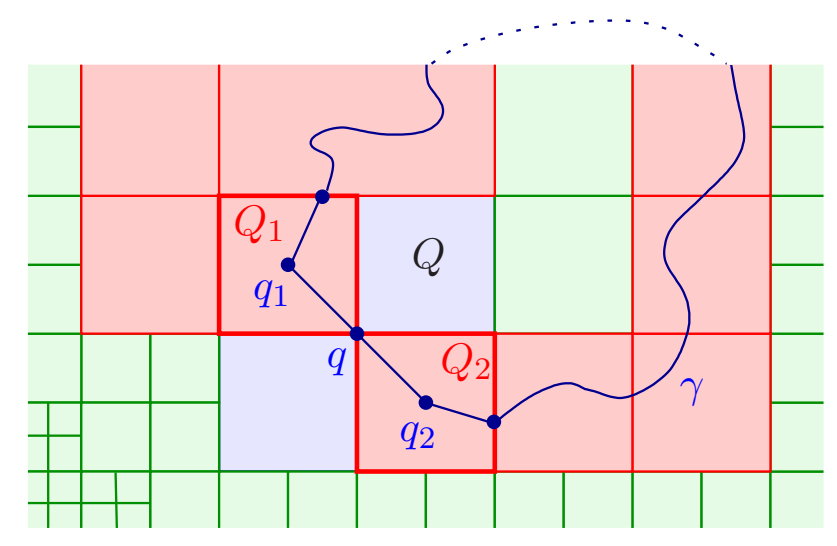

Figure 2. The constructed Jordan curve $\gamma$ in the proof of Lemma $3.1((\mathrm{iii}))$ has in its interior domain a dyadic square $Q$ that also has to be an element of $\mathcal{D}_{n}$.

curve. Let $t_{0}:=\sup \left\{t: \gamma^{\prime}(t) \in Q_{1}\right\}$ and $t_{1}:=\inf \left\{t \geq t_{0}: \gamma^{\prime}(t) \in Q_{2}\right\}$. Define a Jordan curve

$$
\gamma:=\left.\gamma^{1} * \gamma^{2} * \gamma^{\prime}\right|_{\left[t_{0}, t_{1}\right]} * \gamma^{3} * \gamma^{4},
$$

where $\gamma^{1}, \gamma^{2}, \gamma^{3}$ and $\gamma^{4}$ correspond to the line segments $\left[q, q_{1}\right],\left[q_{1}, \gamma_{t_{0}}^{\prime}\right],\left[\gamma_{t_{1}}^{\prime}, q_{2}\right]$ and $\left[q_{2}, q\right]$ respectively. By Jordan curve theorem $\gamma$ divides $\mathbb{R}^{2}$ into two components, one of which is precompact (see Figure 2). Denote the precompact component by $A$.

For small enough ball $B$ around $q$ we have by the definition of $\gamma$ that $B \backslash \gamma$ has exactly two components. Since $\gamma$ is a Jordan curve one of those components has to contain an interior point of $A$ and thus the whole component lies inside $A$. On the other hand that component has to intersect with one of the dyadic squares in $\mathcal{Q}_{n}$ touching both $Q_{1}$ and $Q_{2}$ (but being different from $Q_{1}$ and $Q_{2}$ ). Let $Q \in \mathcal{Q}_{n}$ be that square. Now for all the neighbouring squares $\tilde{Q} \in \mathcal{Q}_{n}$ (except the opposite one) of $Q$ either $\tilde{Q} \cap \gamma([0,1]) \neq \emptyset$ implying that $\tilde{Q} \in \mathcal{D}_{n}$ or $\tilde{Q}$ is in the precompact component of $\mathbb{R}^{2} \backslash \gamma([0,1])$ and thus by simply connectedness $\tilde{Q} \subset \Omega$. Since $Q_{1} \in \mathcal{F}_{n}$, also the opposite square of $Q$ is a subset of $\Omega$. Hence $Q \in \tilde{\mathcal{F}}_{n}$ or $Q \subset Q^{\prime} \in \mathcal{F}_{n-1}$ which is a contradiction. Thus we have proven (iii).

In order to see (iv), suppose that there exists $Q \in \partial \mathcal{D}_{n}$ such that $Q \notin \mathcal{F}_{n}$. Then $Q \in \mathcal{F}_{n, i} \subset \tilde{\mathcal{F}}_{i}$ for some $i<n$. By Property (W4), for all the $Q^{\prime} \in \tilde{\mathcal{F}}$ with $Q^{\prime} \cap Q \neq \emptyset$ we have $Q^{\prime} \in \tilde{\mathcal{F}}_{j}$ for $j \leq i+1 \leq n$. Thus, $Q^{\prime} \subset \bar{D}_{n}$ and $Q \notin \partial \mathcal{D}_{n}$ giving a contradiction.

If property (v) fails for some $Q \in \partial \mathcal{D}_{n}$, then for every $Q^{\prime} \in \tilde{\mathcal{F}}$ with $Q^{\prime} \cap Q \neq \emptyset$ we have $Q^{\prime} \in \tilde{\mathcal{F}}_{i}$ for some $i \leq n$. Thus, again $Q^{\prime} \subset D_{n}$ and $Q \notin \partial \mathcal{D}_{n}$ giving a contradiction.

Finally, we prove property (vi). Since $C_{n}$ is open it suffices to prove that every Jordan curve is loop homotopic to a constant loop. Suppose this is not the case. Then there exists a Jordan curve $\gamma$ that is not homotopic to a constant loop, and a point $x \in \Omega \backslash C_{n}$ that lies inside $\gamma$. In particular there exists $Q \in \mathcal{Q}_{n}$ such that $Q \not \subset D_{n}$ which lies inside $\gamma$ and for which $Q \cap D_{n}$ is an edge of a square. Now by similar argument as in (iii) we conclude that $Q \in \mathcal{D}_{n}$, which is a contradiction. 
The next lemma shows that we can connect the boundary of $D_{n}$ to the boundary of $\Omega$ with a short curve in the complement of $D_{n}$.

Lemma 3.2. For each point $x \in \partial D_{n}$, there exists an injective curve $\gamma:[0,1] \rightarrow \mathbb{R}^{2}$ so that $\gamma(0)=x, \gamma(1) \in \partial \Omega, \gamma(0,1) \subset \Omega \backslash$ int $D_{n}$ and $L(\gamma) \leq 2 \sqrt{2} l(Q)$.

Proof. Let $Q \in \partial \mathcal{D}_{n}$ be such that $x \in Q \cap \partial D_{n}$. By Lemma 3.1 (v) we have that there exists a square $Q^{\prime} \in \mathcal{Q}_{n}$ touching $Q$ at $x$ so that $Q^{\prime} \notin \tilde{\mathcal{F}}_{n}$ and $Q^{\prime} \not \subset \tilde{Q}$ for every $\tilde{Q} \in \tilde{F}_{j}$, $j<n$. Thus, there exists a neighbouring square $Q^{\prime \prime} \in \mathcal{Q}_{n}$ of $Q^{\prime}$ and a point $y \in \partial \Omega \cap Q^{\prime \prime}$. Let $\gamma^{1}$ be a curve corresponding to a line segment connecting $x$ to a point $z \in Q^{\prime} \cap Q^{\prime \prime}$ and let $\gamma^{2}$ be a curve corresponding to a line segment connecting $z$ to $y$. Moreover, let $t_{0}:=\inf \left\{t: \gamma_{t}^{2} \in \partial \Omega\right\}$. Since $\partial \Omega$ is closed, we have that $\gamma_{t_{0}}^{2} \in \partial \Omega$. Define a curve $\gamma:=\left.\gamma^{1} * \gamma^{2}\right|_{\left[0, t_{0}\right]}$. For $\gamma$ we have that $\gamma(0,1) \subset\left(\Omega \backslash\right.$ int $\left.D_{n}\right) \cap\left(Q^{\prime} \cup Q^{\prime \prime}\right), \gamma(0)=x, \gamma(1) \in \partial \Omega$ and $L(\gamma) \leq d\left(Q^{\prime}\right)+d\left(Q^{\prime \prime}\right)=2 \sqrt{2} l(Q)$.

Observe that by Lemma 3.1 (iv) we have $\partial D_{n}=\bigcup_{Q \in \partial \mathcal{D}_{n}}\left(Q \cap \partial D_{n}\right)$. Thus, by Lemma 3.1 (iii) we have that $\partial D_{n}$ is locally homeomorphic to the real line. Since by Lemma 3.1 (vi) $C_{n}$ is simply connected, we have that $\partial D_{n}=\partial C_{n}$ is connected. Hence, $\partial D_{n}$ is a Jordan curve. Thus, we may write

$$
\partial D_{n}=\bigcup_{i=1}^{L_{n}} I_{i},
$$

where $I_{i}=\left[y_{i}, y_{i+1}\right]$ is an edge of a square in $\mathcal{F}_{n}$ with vertices $y_{i}$ and $y_{i+1}$, and $y_{1}=y_{L_{n}+1}$.

For the rest of the paper we fix a constant $M>(4 \sqrt{2}+2)$. However, the following lemma is true for any $M>0$ and with $C$ depending on $M$.

Lemma 3.3. There exists $C \in \mathbb{N}$ so that for any $n \in \mathbb{N}$ and $x, y \in \partial D_{n}$ with $d_{\partial D_{n}}(x, y) \geq$ $2^{-n} C$, and for any $\gamma$ in $\Omega \backslash$ int $D_{n}$ connecting $x$ to $y$ we have that $\gamma \cap\left(\Omega \backslash B\left(x, M 2^{-n}\right)\right) \neq \emptyset$. In particular, $L(\gamma) \geq M 2^{-n}$.

Proof. By taking a slightly larger $C$, namely $C+2$, we may assume that $x=y_{i}$ and $y=y_{j}$ for some $i$ and $j$, where $y_{i}, y_{j}$ are two endpoints of intervals from the collection $\left\{I_{i}\right\}$ forming the boundary as noted above. Moreover, by symmetry we may assume that $i<j$ and $j-i \leq n+1-j$. Since each $I_{i}$ is a side for two squares in $\mathcal{Q}_{n}$, by taking $C$ large enough, we obtain

$$
\left|B\left(x, 2(M+1) 2^{-n}\right)\right|=\pi\left(2(M+1) 2^{-n}\right)^{2}<\frac{1}{2} C\left(2^{-n}\right)^{2} \leq|\bigcup Q|,
$$

where the union is taken over all $Q \in \mathcal{Q}_{n}$ having $I_{m}$ as one of it sides for some $i<$ $m \leq j-1$, and $|\cdot|$ denotes the 2-dimensional Lebesgue measure. Therefore, one of the intervals $I_{m_{1}}$, for $i<m_{1} \leq j-1$, has to intersect with the complement of the ball $B\left(x, 2 M 2^{-n}\right)$. Let $Q_{1}^{\prime} \in \partial \mathcal{D}_{n}$ be the boundary square corresponding to that interval and let $q_{1} \in I_{m_{1}} \backslash B\left(x, 2 M 2^{-n}\right)$. By symmetry, there also exists $Q_{2}^{\prime} \in \partial \mathcal{D}_{n}$ whose side is some $I_{m_{2}}$ with $m_{2} \notin\{i+1, i+1, \ldots, j-1\}$ such that there is $q_{2} \in I_{m_{2}} \backslash B\left(x, 2 M 2^{-n}\right)$.

Suppose now that there exists a curve $\gamma$ in $\Omega \backslash$ int $D_{n}$ joining $x$ to $y$ with $\gamma \subset B\left(x, M 2^{-n}\right)$. We may assume that $\gamma$ is injective, and by compactness that $\gamma(t) \in \Omega \backslash D_{n}$ for every 


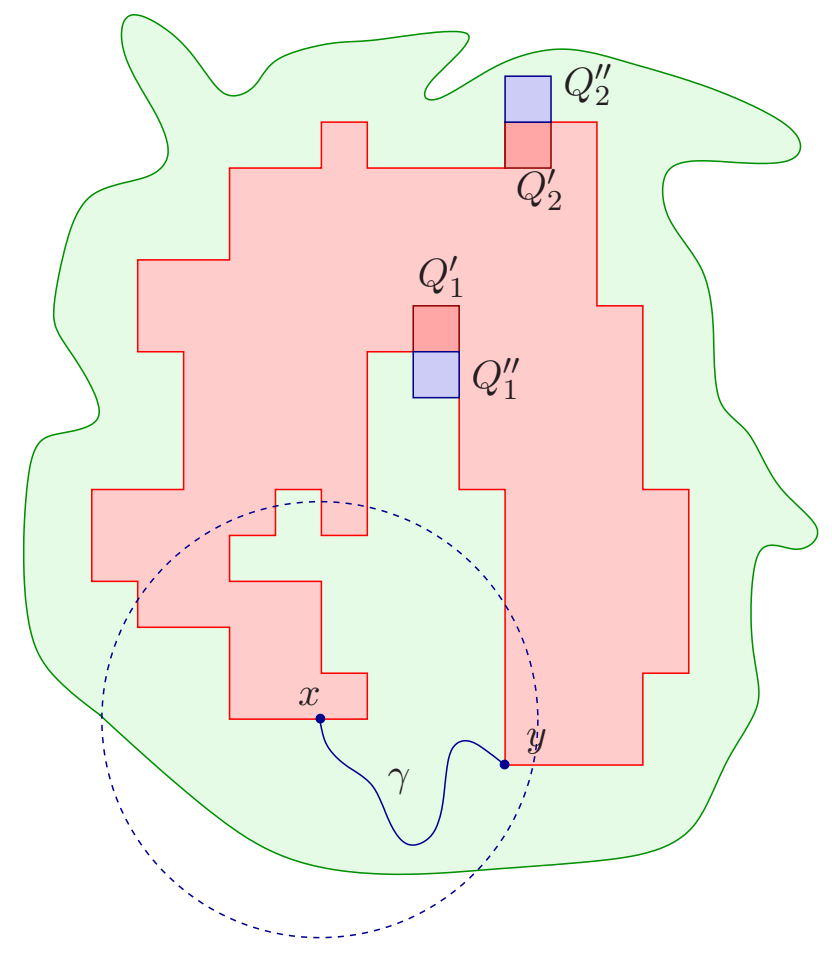

FiguRE 3. In the proof of Lemma 3.3 we assume towards a contradiction that $x$ and $y$ can be connected by a short curve $\gamma$ in $\Omega \backslash D_{n}$. This will imply that one more square in $\mathcal{Q}_{n}$ (here $Q_{1}^{\prime \prime}$ ) will be a subset of $D_{n}$.

$t \in(0,1)$. Then, for $i=1,2$ we have that $B\left(Q_{i}^{\prime}, 2 \sqrt{2} l\left(Q_{i}^{\prime}\right)\right) \subset B\left(q, M 2^{-n}\right)$ and hence $B\left(Q_{i}^{\prime}, 2 \sqrt{2} l\left(Q^{\prime}\right)\right) \cap \gamma=\emptyset$. Now by definition of $Q_{i}^{\prime}$ there is a neighbouring square $Q_{i}^{\prime \prime} \in \mathcal{Q}_{n}$ of $Q_{i}^{\prime}$ which is not a subset of $D_{n}$, see Figure 3 . We claim that either $Q_{1}^{\prime \prime}$ or $Q_{2}^{\prime \prime}$ lies inside the Jordan curve $\gamma^{\prime}$ obtained by concatenating the curve $\gamma$ and the part of the boundary, denoted by $\gamma^{\prime \prime}$, obtained from the intervals $\left\{I_{h}\right\}_{h=i}^{j-1}$, or by concatenating $\gamma$ and $\partial D_{n} \backslash \gamma^{\prime \prime}$.

This can be seen in the following way. Consider $\Omega \stackrel{h}{\rightarrow} \mathbb{R}^{2} \hookrightarrow \mathbb{S}^{2}$, where $h$ is a homeomorphism and the inclusion $\mathbb{R}^{2} \hookrightarrow \mathbb{S}^{2}$ is the inverse of the stereographic projection. Under this composite map $\mathbb{S}^{2} \backslash D_{n}$ is a simply connected domain. Hence, by Lemma $2.1\left(\mathbb{S}^{2} \backslash D_{n}\right) \backslash \gamma$ has exactly two components whose boundaries are the two connected components of $\partial D_{n} \backslash \gamma$ together with $\gamma$. Thus, $\left(\Omega \backslash D_{n}\right) \backslash \gamma=\left(\mathbb{S}^{2} \backslash D_{n}\right) \backslash \gamma$ has exactly two components. Since $\partial Q_{1}^{\prime \prime} \cap \partial D_{n}$ and $\partial Q_{2}^{\prime \prime} \cap \partial D_{n}$ are in two different connected components of $\partial D_{n} \backslash \gamma$, we conclude that $Q_{1}^{\prime \prime}$ and $Q_{2}^{\prime \prime}$ are in different components of $\left(\Omega \backslash D_{n}\right) \backslash \gamma$. We denote the $Q_{i}^{\prime \prime}$ that lies inside the Jordan curve by $Q^{\prime \prime}$.

Since $Q^{\prime \prime} \subset B\left(Q^{\prime}, \sqrt{2} l\left(Q^{\prime}\right)\right)$, we have that every neighbouring square of $Q^{\prime \prime}$ either lies inside $\gamma^{\prime}$ or is an element of $\partial \mathcal{D}_{n}$. In particular, by the simply connectedness of $\Omega$ they all are subsets of $\Omega$. Hence, $Q^{\prime \prime} \subset D_{n}$ which is a contradiction. Thus, we have proven that $\gamma \cap\left(\Omega \backslash B\left(x, M 2^{-n}\right)\right) \neq \emptyset$. 


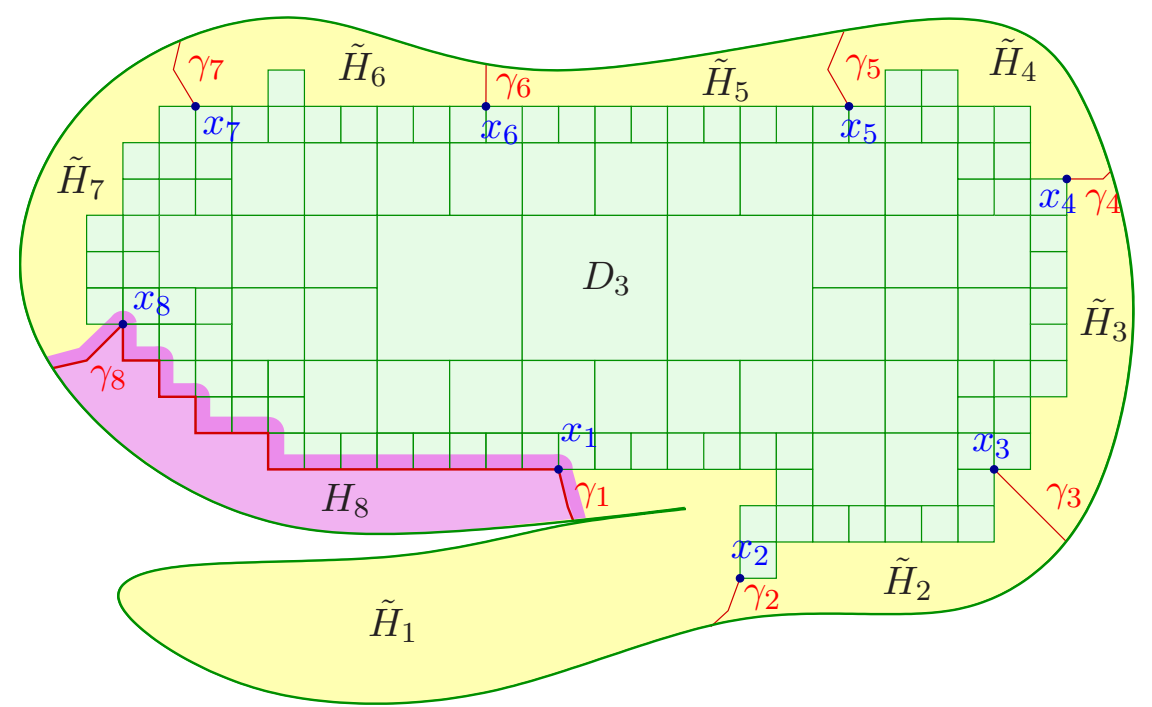

Figure 4. Here the domain $\Omega$ is decomposed into the core part $D_{3}$ and eight boundary parts $\tilde{H}_{i}$. A neighbourhood $H_{8}$ of $\tilde{H}_{8}$ is also illustrated.

Let us now partition $\Omega \backslash D_{n}$ in the following way. Recall $(3.1)$. Notice that for large enough $n$ we have that $L_{n} \geq 2 C$. Define $x_{1}:=y_{1}$ and then $x_{m}:=y_{(m-1) C}$ until $L_{n}+1-(m-$ 1) $C<2 C$. Notice that for every $i \neq j$ we have $d_{\partial D_{n}}\left(x_{i}, x_{j}\right) \geq 2^{-n} C$. We now partition the set $\Omega \backslash D_{n}$ up to Lebesgue measure zero into connected sets $\left\{\tilde{H}_{j}\right\}_{j=1}^{m}$ where $\tilde{H}_{j}$ is the open set bounded by $\gamma_{j}, \gamma_{j+1}$ given by Lemma 3.2 for points $x_{j}$ and $x_{j+1}$, and $J_{j}:=\bigcup_{i=C j}^{C(j+1)} I_{i}$ (with interior in $\Omega \backslash D_{n}$ ). This partition is well defined by Lemma 2.1. Notice that since $L\left(\gamma_{i}\right) \leq M$ for all $i$, we have that $\gamma_{i} \cap \gamma_{j}=\emptyset$ for all $i \neq j$. Let us define $H_{j}$ as the connected component containing $\tilde{H}_{j}$ of the set $\Omega \cap\left(\tilde{H}_{j} \cup B_{\mathbb{R}^{2}}\left(\gamma_{j} \cup \gamma_{j+1} \cup J_{j}, \delta\right)\right)$, where $\delta=2^{-n-3}$. See Figure 4 for an illustration of the decomposition. Although the decomposition depends on $n$, for simplicity we do not display the dependence in the notation. A crucial property of our decomposition is the following lemma.

Lemma 3.4. We have $H_{j} \cap H_{i} \neq \emptyset$, if and only if $|i-j| \leq 1$ in a cyclic manner.

Proof. Trivially $\gamma_{i+1} \in H_{i} \cap H_{i+1}$. Thus, we only need to show that $H_{j} \cap H_{i} \neq \emptyset$ implies $|i-j| \leq 1$. We may assume that $i \neq j$. Let $x \in H_{i} \cap H_{j}$.

Suppose first that $x \in \tilde{H}_{i}$. Then, by (path) connectedness of $H_{j}$ there exists a path $\gamma$ in $H_{j}$ from $x$ to $\tilde{H}_{j}$. Let

$$
t_{0}:=\inf \left\{t \in[0,1]: \gamma(t) \notin \tilde{H}_{i}\right\} .
$$

Then, $\gamma\left(t_{0}\right) \notin \tilde{H}_{i}$ but $\gamma(t) \in H_{i} \cap H_{j}$. Thus it suffices to consider the case when $x \notin \tilde{H}_{i} \cup \tilde{H}_{j}$.

Suppose now that $x \in D_{n}$. Since $\delta<\frac{2^{-n}}{2}$, we have that $x \in Q$ for some $Q \in \partial \mathcal{D}_{n}$. Then, there are neighbouring squares $Q_{i}, Q_{j} \in \mathcal{Q}_{n}$ of $Q$ for which $Q_{i} \cap \tilde{H}_{i} \neq \emptyset$ and $Q_{j} \cap \tilde{H}_{j} \neq \emptyset$. Since $\delta$ is small, we may choose the $Q_{i}, Q_{j}$ so that $Q_{i} \cap Q_{j} \neq \emptyset$. If $Q_{i}=Q_{j}$ or if $Q_{i}$ and $Q_{j}$ 
have a common edge, then there is a curve $\gamma^{\prime}$ in $Q_{i} \cup Q_{j}$ from $\tilde{H}_{i}$ to $\tilde{H}_{j}$ with $L\left(\gamma^{\prime}\right)<2 \delta$. If $Q_{i} \cap Q_{j}$ is a singleton, then by Lemma 3.1 (iii) the neighbouring square $Q^{\prime} \neq Q$ of both $Q_{i}$ and $Q_{j}$ lies in $\Omega \backslash$ int $D_{n}$. Indeed, if this were not the case, then $Q^{\prime}, Q \in \mathcal{F}_{n}$ and $Q^{\prime} \cap Q$ is a singleton, implying that $Q_{i} \in \mathcal{D}_{n}$ or $Q_{j} \in \mathcal{D}_{n}$. Thus, there exists a curve $\gamma^{\prime}$ in $\Omega \backslash D_{n}$ joining $Q_{i}$ and $Q_{j}$ with $L\left(\gamma^{\prime}\right)<4 \delta$.

Now, we have $Q_{i} \cap J_{i} \neq \emptyset$ or $Q_{i} \cap\left(\gamma_{i} \cup \gamma_{i+1}\right) \neq \emptyset$. Notice that $\gamma_{i} \cap J_{i} \neq \emptyset \neq \gamma_{i+1} \cap J_{i}$. By Lemma 3.2 we have $\max \left(l\left(\gamma_{i}\right), l\left(\gamma_{i+1}\right)\right) \leq 2 \sqrt{2} \cdot 2^{-n}$. Combining these observations with the analogous ones for $Q_{j}$, we have that $J_{i}$ and $J_{j}$ can be connected by a curve in $\Omega \backslash D_{k}$ with length less than $4 \delta+4 \sqrt{2} \cdot 2^{-n}<2^{-n} M$. Hence, we have by Lemma 3.3 that $\operatorname{dist}_{\partial D_{n}}\left(J_{i}, J_{j}\right) \leq C$. Thus, $|i-j| \leq 1$ in cyclical manner.

We are left with the case where $x \in \Omega \backslash\left(D_{n} \cup \tilde{H}_{i} \cup \tilde{H}_{j}\right)$. By definition we have that $B\left(D_{n}, 2 \delta\right) \subset \Omega$. Thus, if $\operatorname{dist}\left(x, J_{i}\right)<\delta$, we may join $x$ to $J_{i}$ by a curve in $\Omega \backslash$ int $D_{n}$ with length less than $\delta$. If $\operatorname{dist}\left(x, J_{i}\right) \geq \delta$, then $x \in B\left(\gamma_{m}, \delta\right)$, where $m \in\{i, i+1\}$. By path connectedness of $H_{i}$ there is a curve $\gamma$ in $H_{i}$ connecting $x$ to $\gamma_{i} \cup \gamma_{i+1} \cup J_{i}$. We want to prove that $x$ can be joined to $\gamma_{m}$ in the $\delta$-neighbourhood of $\gamma_{m}$. If (a subcurve of) $\gamma$ is not such a curve, then we may define

$$
t_{0}:=\inf \left\{t \in[0,1]: \gamma(t) \in B\left(D_{n}, \delta\right)\right\} .
$$

Then, $\left.\gamma\right|_{\left[0, t_{0}\right]} \subset B\left(\gamma_{m}, \delta\right)$. Therefore, there exists a point $y \in \gamma_{m}$ with $d\left(\gamma\left(t_{0}\right), y\right)<\delta$. In particular, the line segment $\left[\gamma\left(t_{0}\right), y\right]$ lies in $\left(\Omega \backslash D_{n}\right) \cap B\left(\gamma_{m}, \delta\right)$ and thus we have proven that there exists a curve $\gamma^{\prime}$ in $\left(\Omega \backslash D_{n}\right) \cap B\left(\gamma_{m}, \delta\right)$ connecting $x$ to $\gamma_{m}$. By the definition of $\gamma_{m}$ we have that $\gamma^{\prime} \subset B\left(\gamma_{m}(0), 2 \sqrt{2} \cdot 2^{-n}+\delta\right)$. By the same argument for $j$ we conclude that $J_{i}$ and $J_{j}$ can actually be connected by a curve $\gamma$ in $\left(\Omega \backslash \operatorname{int} D_{n}\right) \cap B\left(\gamma(0), 4 \sqrt{2} \cdot 2^{-n}+2 \delta\right)$. Hence, by Lemma $3.3 \operatorname{dist}_{\partial D_{n}}\left(J_{i}, J_{j}\right)<C$, and thus $|i-j| \leq 1$ in cyclical manner.

\section{Approximation}

In this section we finish the proof of Theorem 1.1 by making a partition of unity using the decomposition of $\Omega$ constructed in Section 3 and by approximating a given function by polynomials in this decomposition. Recall that our aim is to show that for any $u \in L^{k, p}(\Omega)$ and $\epsilon>0$ there exists a function $u_{\epsilon} \in W^{k, \infty}(\Omega) \cap C^{\infty}(\Omega)$ with $\left\|\nabla^{k} u-\nabla^{k} u_{\epsilon}\right\|_{L^{p}(\Omega)} \lesssim \epsilon$. By noting that $L^{k, p}(\Omega) \cap C^{\infty}(\Omega)$ is dense in $L^{k, p}(\Omega)$ we may assume that function $u \in$ $L^{k, p}(\Omega) \cap C^{\infty}(\Omega)$. From now on, let $u$ and $\epsilon>0$ be fixed.

Using the notation from Section 3 , we write the domain $\Omega$ as the union of the core part $D_{n}$ and the boundary regions $\left\{H_{i}\right\}_{i=1}^{l}$. For each $\tilde{H}_{i}$ we let $\mathcal{I}_{i}$ be the collection of squares $Q$ in $\partial \mathcal{D}_{n}$ such that $Q \cap \tilde{H}_{i} \neq \emptyset$, and note that the cardinality of $\mathcal{I}_{i}$ is bounded in number independently of $n$. We need to decide what polynomial to attach to each set $H_{i}$. For this purpose, for each $1 \leq i \leq l$ we assign a square $Q_{i} \in \mathcal{I}_{i}$. We call $Q_{i}$ the associated square of $H_{i}$.

Given $Q \in \mathcal{I}_{i}$ we set $\mathcal{P}_{Q}:=\bigcup_{j=i-1}^{i+1}\left\{Q^{\prime} \in \mathcal{I}_{j}\right\}$, which is a collection of squares from a suitable neighbourhood of $Q$.

Recall the approximating polynomials $P_{Q}$ introduced in Section 2.2. We abbreviate $P_{i}=P_{Q_{i}}$ for the associated squares $Q_{i}$. 
We make a smooth partition of unity by using a Euclidean mollification. (Compare to [14] where the inner distance in $\Omega$ was used for the mollification.) Let $\rho_{r}$ denote a standard Euclidean mollifier supported in $B(0, r)$. We start with a collection of functions $\left\{\tilde{\psi}_{i}\right\}_{i=0}^{l}$, where $\tilde{\psi}_{0}=\chi_{D_{n}} * \rho_{2^{-n-5}}$ and $\tilde{\psi}_{i}=\left.\left(\chi_{\tilde{H}_{i}} * \rho_{2^{-n-5}}\right)\right|_{H_{i}}$ for $i \geq 1$. Using this we obtain a partition of unity $\left\{\psi_{i}\right\}_{i=0}^{l}$ by setting $\psi_{i}=\tilde{\psi}_{i} / \sum_{j=0}^{l} \tilde{\psi}_{j}$.

Now the partition of unity $\left\{\psi_{i}\right\}_{i=0}^{l}$ satisfies the following.

(1) The function $\psi_{0}$ is supported in $B\left(D_{n}, \frac{2^{-n}}{10}\right)$.

(2) For $i \geq 1$ the function $\psi_{i}$ is supported in $H_{i}$.

(3) For all $i, 0 \leq \psi_{i} \leq 1$.

(4) $\sum \psi_{i} \equiv 1$ on $\Omega$.

(5) For all $i,\left|\nabla^{\alpha} \psi_{i}\right| \leq C_{\alpha} 2^{-n|\alpha|}$ for all multi-indeces $\alpha$.

We will fix $n$ later such that for the function $u_{\epsilon}$ defined as

$$
u_{\epsilon}(x):=u(x) \psi_{0}(x)+\sum_{i=1}^{l} \psi_{i}(x) P_{i}(x)
$$

for $x \in \Omega$, we have

$$
\left\|\nabla^{k} u-\nabla^{k} u_{\epsilon}\right\|_{L^{p}(\Omega)}<C \epsilon .
$$

Note that $u_{\epsilon}=u$ on $D_{n-1}$; indeed $D_{n-1} \cap \psi_{i}=\emptyset$ for $i \geq 1$, see Lemma 3.1 (iv)

First of all, we consider only $n$ large enough so that

$$
\left\|\nabla^{k} u\right\|_{L^{p}\left(\Omega \backslash D_{n-1}\right)} \leq \epsilon
$$

Now, we need to show that $n$ can actually be chosen large enough so that also

$$
\left\|\nabla^{k} u_{\epsilon}\right\|_{L^{p}\left(\Omega \backslash D_{n-1}\right)} \leq C \epsilon .
$$

So, we compute for $Q \in \mathcal{I}_{i}$ and $|\alpha|=k$

$$
\begin{aligned}
\left\|\nabla^{\alpha} u_{\epsilon}\right\|_{L^{p}(Q)} & \leq \sum_{\beta \leq \alpha}\left(\int_{Q}\left|\nabla^{\beta} u-\nabla^{\beta} P_{i}(x)\right|^{p}\left|\nabla^{\alpha-\beta} \psi_{0}(x)\right|^{p} \mathrm{~d} x\right)^{1 / p} \\
& +\sum_{\beta \leq \alpha} \sum_{j}\left(\int_{Q}\left|\nabla^{\beta} P_{j}(x)-\nabla^{\beta} P_{i}(x)\right|^{p}\left|\nabla^{\alpha-\beta} \psi_{j}(x)\right|^{p} \mathrm{~d} x\right)^{1 / p} \\
& =: A_{1}+A_{2},
\end{aligned}
$$

where $A_{1}$ and $A_{2}$ are the first and second terms on the right hand side of the inequality and we used that for $\beta<\alpha, \sum_{j} \nabla^{\alpha-\beta} \psi_{j}=0$ and order of $P_{i}$ is at most $k-1$. We first 
estimate $A_{1}$ as

$$
\begin{aligned}
A_{1} & \lesssim \sum_{\beta \leq \alpha} 2^{n(|\alpha|-|\beta|)}\left\|\nabla^{\beta} u-\nabla^{\beta} P_{i}\right\|_{L^{p}(Q)} \\
& \lesssim \sum_{\beta \leq \alpha} 2^{n(|\alpha|-|\beta|)}\left(\left\|\nabla^{\beta} P_{i}-\nabla^{\beta} P_{Q}\right\|_{L^{p}(Q)}+\left\|\nabla^{\beta} u-\nabla^{\beta} P_{Q}\right\|_{L^{p}(Q)}\right) \\
& \lesssim \sum_{\beta \leq \alpha} 2^{n(|\alpha|-|\beta|)} 2^{n(|\beta|-k)}\left\|\nabla^{k} u\right\|_{L^{p}(\cup \tilde{Q})} \\
& \lesssim\left\|\nabla^{k} u\right\|_{L^{p}(\cup \tilde{Q})},
\end{aligned}
$$

where in the third inequality we used that $Q_{i}$ (associated square of $\tilde{H}_{i}$ ) and $Q$ may be joined by a chain of bounded number of squares from $\mathcal{I}_{i}$ by our construction, and therefore we may apply Lemma 2.5. Similarly we estimate $A_{2}$ as

$$
\begin{aligned}
A_{2} & \lesssim \sum_{\beta \leq \alpha} \sum_{j=i-1}^{i+1}\left(\int_{Q}\left|\nabla^{\beta} P_{j}(x)-\nabla^{\beta} P_{i}(x)\right|^{p}\left|\nabla^{\alpha-\beta} \psi_{j}(x)\right|^{p} \mathrm{~d} x\right)^{1 / p} \\
& \lesssim \sum_{\beta \leq \alpha} 2^{n(|\alpha|-|\beta|)} \sum_{j=i-1}^{i+1}\left(\left\|\nabla^{\beta} P_{j}-\nabla^{\beta} P_{Q}\right\|_{L^{p}(Q)}+\left\|\nabla^{\beta} P_{i}-\nabla^{\beta} P_{Q}\right\|_{L^{p}(Q)}\right) \\
& \lesssim \sum_{\beta \leq \alpha} 2^{n(|\alpha|-|\beta|)} 2^{n(|\beta|-k)}\left\|\nabla^{k} u\right\|_{L^{p}(\cup \tilde{Q})} \\
& \lesssim\left\|\nabla^{k} u\right\|_{L^{p}(\cup \tilde{Q})}
\end{aligned}
$$

where again in the second inequality we used that if $\psi_{j}(x) \neq 0$ for $x \in Q \in \mathcal{I}_{i}$ then by our construction $Q_{j}$ and $Q$ can be joined by a chain of bounded number of squares as $j$ is either $i-1, i$ or $i+1$ (cyclically); and therefore we can apply Lemma 2.5.

For $Q \in \tilde{\mathcal{F}} \backslash \mathcal{D}_{n}$ such that $Q \cap \operatorname{spt}\left(\psi_{0}\right) \neq \emptyset$, we assign to $Q$ a square $Q^{\prime} \in \mathcal{I}_{i}$, such that $Q \cap Q^{\prime} \neq \emptyset$. Note that such a square $Q^{\prime}$ exists by our construction. Then $Q$ and $Q^{\prime}$ can be joined by a chain of bounded (by an absolute constant) number of squares from $\mathcal{D}_{n+1}$. We choose such a chain for $Q$ and denote it by $\mathcal{B}_{Q}$. We also set

$$
\mathcal{J}_{n}:=\left\{Q \in \tilde{\mathcal{F}} \backslash \mathcal{D}_{n}: Q \cap \operatorname{spt}\left(\psi_{0}\right) \neq \emptyset\right\} .
$$

We estimate using Lemma 2.5 exactly as above (see (4.2)) to obtain for $|\alpha|=k$

$$
\begin{aligned}
\left\|\nabla^{\alpha} u_{\epsilon}\right\|_{L^{p}(Q)} & \leq \sum_{\beta \leq \alpha}\left(\int_{Q}\left|\nabla^{\beta} u-\nabla^{\beta} P_{Q}(x)\right|^{p}\left|\nabla^{\alpha-\beta} \psi_{0}(x)\right|^{p} \mathrm{~d} x\right)^{1 / p} \\
& +\sum_{\beta \leq \alpha} \sum_{j}\left(\int_{Q}\left|\nabla^{\beta} P_{j}(x)-\nabla^{\beta} P_{Q}(x)\right|^{p}\left|\nabla^{\alpha-\beta} \psi_{j}(x)\right|^{p} \mathrm{~d} x\right)^{1 / p} \\
& =: B_{1}+B_{2} .
\end{aligned}
$$


Again, we estimate separately,

$$
\begin{aligned}
B_{1} & \lesssim \sum_{\beta \leq \alpha} 2^{n(|\alpha|-|\beta|)}\left\|\nabla^{\beta} u-\nabla^{\beta} P_{Q}\right\|_{L^{p}(Q)} \\
& \lesssim\left\|\nabla^{k} u\right\|_{L^{p}(Q)}
\end{aligned}
$$

and

$$
\begin{aligned}
& B_{2} \lesssim \sum_{\beta \leq \alpha} \sum_{j=i-1}^{i+1}\left(\int_{Q}\left|\nabla^{\beta} P_{j}(x)-\nabla^{\beta} P_{Q}(x)\right|^{p}\left|\nabla^{\alpha-\beta} \psi_{j}(x)\right|^{p} \mathrm{~d} x\right)^{1 / p} \\
& \lesssim \sum_{\beta \leq \alpha} 2^{n(|\alpha|-|\beta|)} \sum_{j=i-1}^{i+1}\left(\left\|\nabla^{\beta} P_{j}-\nabla^{\beta} P_{Q^{\prime}}\right\|_{L^{p}(Q)}+\left\|\nabla^{\beta} P_{Q^{\prime}}-\nabla^{\beta} P_{Q}\right\|_{L^{p}(Q)}\right)
\end{aligned}
$$

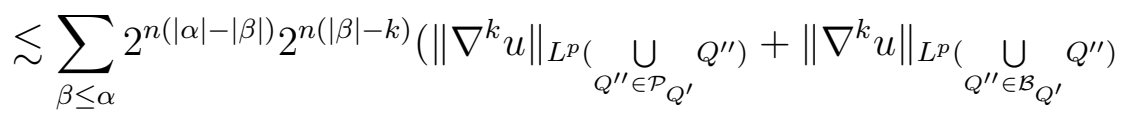

$$
\begin{aligned}
& \lesssim\left\|\nabla^{k} u\right\|_{L^{p}\left(\underset{Q^{\prime \prime} \in \mathcal{P}_{Q^{\prime}} \cup \mathcal{B}_{Q^{\prime}}}{\cup} Q^{\prime \prime}\right)}
\end{aligned}
$$

Next we note that $\nabla^{k} u_{\epsilon} \equiv 0$ in $\tilde{H}_{i} \backslash \cup_{j \neq i} \operatorname{spt}\left(\psi_{j}\right)$ and we compute for $|\alpha|=k$

$$
\begin{aligned}
\left\|\nabla^{\alpha} u_{\epsilon}\right\|_{L^{p}\left(H_{i}\right)} & \leq \sum_{Q \in \tilde{Q}_{i}}\left\|\nabla^{\alpha} u_{\epsilon}\right\|_{L^{p}(Q)}+\sum_{Q \in \mathcal{J}_{n}, Q \cap H_{i} \neq \emptyset}\left\|\nabla^{\alpha} u_{\epsilon}\right\|_{L^{p}(Q)} \\
& \left.+\sum_{j=i-1}^{i+1}\left\|\nabla^{\alpha} u_{\epsilon}\right\|_{L^{p}\left(\left(\operatorname{spt}\left(\psi_{j}\right) \cap \operatorname{spt}\left(\psi_{i}\right)\right) \backslash\right.} \underset{Q^{\prime \prime} \in \partial \mathcal{D}_{n} \cap \mathcal{J}_{n}}{\cup} Q^{\prime \prime}\right)
\end{aligned}
$$

The terms in the first and second summands have been estimated earlier. Denoting $H_{i}^{\prime}:=$ $\left(\cup_{j=i-1}^{i+1} \operatorname{spt}\left(\psi_{j}\right) \cap \operatorname{spt}\left(\psi_{i}\right)\right) \backslash \bigcup_{Q^{\prime \prime} \in \partial \mathcal{D}_{n} \cap \mathcal{J}_{n}} Q^{\prime \prime}$, we estimate now the third one;

$$
\begin{aligned}
& \left\|\nabla^{\alpha} u_{\epsilon}\right\|_{L^{p}\left(H_{i}^{\prime}\right)} \lesssim \sum_{\beta \leq \alpha} 2^{n(|\alpha|-|\beta|)} \sum_{j=i-1}^{i+1}\left\|\nabla^{\beta} P_{j}-\nabla^{\beta} P_{i}\right\|_{L^{p}\left(H_{i}^{\prime}\right)} \\
& \lesssim \sum_{\beta \leq \alpha} 2^{n(|\alpha|-|\beta|)}\left(\left\|\nabla^{\beta} P_{j}-\nabla^{\beta} P_{i}\right\|_{L^{p}\left(Q_{i}\right)}\right. \\
& \left.\lesssim \sum_{\beta \leq \alpha} 2^{n(|\alpha|-|\beta|)} 2^{n(|\beta|-k)}\left\|\nabla^{k} u\right\|_{L^{p}(} \underset{Q^{\prime} \in \mathcal{P}_{Q_{i}}}{\cup} Q^{\prime}\right) \\
& \lesssim\left\|\nabla^{k} u\right\|_{L^{p}\left(\underset{Q^{\prime} \in \mathcal{P}_{Q_{i}}}{\cup} Q^{\prime}\right)},
\end{aligned}
$$

where we used the facts that for $\beta<\alpha, \nabla^{\alpha-\beta} \sum_{j}\left(\psi_{j}\right)=0$ and $\psi_{0} \equiv 0$ in $H_{i}^{\prime}$ in the first inequality, Lemma 2.4 in the second inequality since $H_{i}^{\prime} \subset C Q_{i}$ for some absolute constant $C$ coming from Lemma 3.2 and in the third inequality we used Lemma 2.5. 
Remark 4.1. Note that for each $Q \in \mathcal{I}_{i}$ we have $\mathcal{P}_{Q}=\mathcal{P}_{Q_{i}}$ where $Q_{i}$ is the associated square of $\tilde{H}_{i}$. We note that any $Q^{\prime} \in \partial \mathcal{D}_{n}$ occurs in at most three distinct collections $\mathcal{P}_{Q_{i}}$. Moreover any $Q \in \mathcal{D}_{n+1}$ appears in only a bounded number of the collections $\mathcal{B}_{Q^{\prime \prime}}$, where $Q^{\prime \prime} \in \mathcal{J}_{n}$. In particular, any $Q^{\prime} \in \partial \mathcal{D}_{n}$ appears in only a bounded number of the collections $\mathcal{B}_{Q^{\prime \prime}}$, where $Q^{\prime \prime} \in \mathcal{J}_{n}$. The bounds are provided by absolute constants coming from volume comparison.

Now it follows from equations (4.3), 4.4), 4.5), 4.6) and (4.7) that

$$
\begin{aligned}
& \left\|\nabla^{\alpha} u_{\epsilon}\right\|_{L^{p}\left(\Omega \backslash C_{n}\right)} \lesssim \sum_{i}\left\|\nabla^{k} u\right\|_{L^{p}\left(H_{i}\right)}+\left\|\nabla^{k} u\right\|_{L^{p}\left(\bigcup_{Q \in \partial \mathcal{D}_{n}} Q\right)}
\end{aligned}
$$

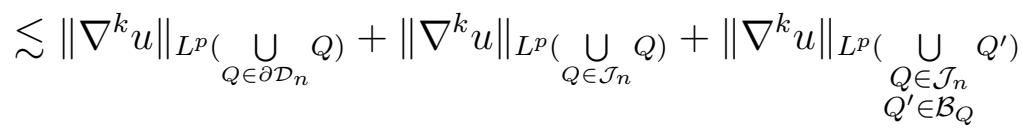

when $|\alpha|=k$. By Remark 4.1 we may choose $n$ such that

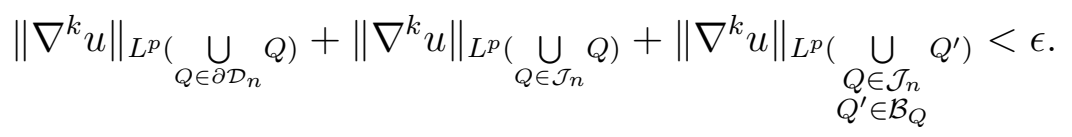

Then, the claim follows from 4.1) and 4.8.

Remark 4.2. We note that when $k=1$ we may take the function to be smooth as well as bounded for showing the density of $W^{1, \infty}(\Omega)$ in $W^{1, p}(\Omega)$. This is because truncations approximate the functions in $W^{1, p}(\Omega)$. This allows us to also approximate the $L^{p}$ norm of $u$. Indeed, let $u \in W^{1, p}(\Omega) \cap C^{\infty}(\Omega) \cap L^{\infty}(\Omega)$ such that $\|u\|_{L^{\infty}} \leq M$. Decompose the domain as in the above construction; then choose $n$ large enough such that $\|u\|_{W^{1, p}\left(\Omega \backslash D_{n-1}\right)} \leq \epsilon$ and $M\left|\Omega \backslash D_{n-1}\right|<\epsilon$. Then it follows from estimates in the proof that the function $u_{\epsilon}$ defined as above approximates $u$ in $W^{1, p}(\Omega)$ with error given by $\epsilon$. This conclusion is the content of [14].

Finally, let us show how the smooth approximation in Jordan domains is done.

Proof of Corollary 1.3. The argument we need follows the one used to prove [14, Corollary 1.2]. As in [14], given a bounded Jordan domain we approximate it from outside by a nested sequence of Lipschitz and simply connected domains $G_{s}$ which are obtained for example by taking the complement of the unbounded connected component of the union of Whitney squares larger than $2^{-s}$ from the Whitney decomposition of the complementary Jordan domain of $\Omega$.

Then, we note that for given $n$, taking $s_{n}$ large enough, we have that the squares in $\partial \mathcal{D}_{n}$ are Whitney type sets in $G_{s_{n}}$, meaning they have diameters comparable to the distance from the boundary of $G_{s_{n}}$.

Note that $G_{s_{n}} \subset B\left(\Omega, 2^{-s_{n}+5}\right)$ are simply connected. Now the set $G_{s_{n}} \backslash \bar{C}_{n}$ (recall that $C_{n}$ is a suitable connected component of the interior of the union of the Whitney squares of scale less than $2^{-n}$ ) can be decomposed in the same way as $\Omega \backslash \bar{C}_{n}$ was decomposed into the sets $\tilde{H}_{i}$ in Section 3 . 
We may then follow the argument used in the proof of Theorem 1.1 to obtain an approximating sequence of functions $u_{n}$ in $G_{s_{n}}$ which are in the space $W^{k, \infty}\left(G_{s_{n}}\right) \cap L^{k, p}\left(G_{s_{n}}\right) \cap$ $C^{\infty}\left(G_{s_{n}}\right)$. By multiplying with a smooth cut-off function that is 1 on $\Omega$ and compactly supported in $G_{s_{n}}$, we obtain a sequence of global smooth functions having the desired properties.

\section{REFERENCES}

1. Charles J. Amick, Approximation by smooth functions in Sobolev spaces, Bull. London Math. Soc. 11 (1979), no. 1, 37-40. MR 535794

2. Christopher J. Bishop, A counterexample concerning smooth approximation, Proc. Amer. Math. Soc. 124 (1996), no. 10, 3131-3134. MR 1328340

3. Stephen M. Buckley and Pekka Koskela, Criteria for imbeddings of Sobolev-Poincaré type, Internat. Math. Res. Notices (1996), no. 18, 881-901. MR 1420554

4. Alessandro Giacomini and Paola Trebeschi, A density result for Sobolev spaces in dimension two, and applications to stability of nonlinear Neumann problems, J. Differential Equations 237 (2007), no. 1, 27-60. MR 2327726

5. V. M. Gol'dshteĭn and Yu. G. Reshetnyak, Quasiconformal mappings and Sobolev spaces, Mathematics and its Applications (Soviet Series), vol. 54, Kluwer Academic Publishers Group, Dordrecht, 1990, Translated and revised from the 1983 Russian original, Translated by O. Korneeva. MR 1136035

6. V. M. Gol'dsteı̆n, T. G. Latfullin, and S. K. Vodop'janov, A criterion for the extension of functions of the class $L_{2}^{1}$ from unbounded plane domains, Sibirsk. Mat. Zh. 20 (1979), no. 2, 416-419, 464. MR 530508

7. Vladimir Gol'dsteĭn and Serge Vodop'anov, Prolongement de fonctions différentiables hors de domaines plans, C. R. Acad. Sci. Paris Sér. I Math. 293 (1981), no. 12, 581-584. MR 647686

8. Peter W. Jones, Quasiconformal mappings and extendability of functions in Sobolev spaces, Acta Math. 147 (1981), no. 1-2, 71-88. MR 631089

9. Torbjörn Kolsrud, Approximation by smooth functions in Sobolev spaces, a counterexample, Bull. London Math. Soc. 13 (1981), no. 2, 167-169. MR 608104

10. Pekka Koskela, Extensions and imbeddings, J. Funct. Anal. 159 (1998), no. 2, 369-383. MR 1658090

11. __ Removable sets for Sobolev spaces, Ark. Mat. 37 (1999), no. 2, 291-304. MR 1714767

12. Pekka Koskela, Tapio Rajala, and Yi Ru-Ya Zhang, A geometric characterization of planar sobolev extension domains, Preprint.

13. _ A density problem for Sobolev spaces on Gromov hyperbolic domains, Nonlinear Anal. 154 (2017), 189-209. MR 3614650

14. Pekka Koskela and Yi Ru-Ya Zhang, A density problem for Sobolev spaces on planar domains, Arch. Ration. Mech. Anal. 222 (2016), no. 1, 1-14. MR 3519964

15. John L. Lewis, Approximation of Sobolev functions in Jordan domains, Ark. Mat. 25 (1987), no. 2, 255-264. MR 923410

16. Norman G. Meyers and James Serrin, $H=W$, Proc. Nat. Acad. Sci. U.S.A. 51 (1964), 1055-1056. MR 0164252

17. M. H. A. Newman, Elements of the topology of plane sets of points, Cambridge, At the University Press, 1951, 2nd ed. MR 0044820

18. Anthony G. O'Farrell, An example on Sobolev space approximation, Bull. London Math. Soc. 29 (1997), no. 4, 470-474. MR 1446566

19. Pavel Shvartsman, On Sobolev extension domains in $\mathbb{R}^{n}$, J. Funct. Anal. 258 (2010), no. 7, 2205-2245. MR 2584745

20. Wayne Smith, Alexander Stanoyevitch, and David A. Stegenga, Smooth approximation of Sobolev functions on planar domains, J. London Math. Soc. (2) 49 (1994), no. 2, 309-330. MR 1260115 
21. Wayne Smith and David A. Stegenga, Hölder domains and Poincaré domains, Trans. Amer. Math. Soc. 319 (1990), no. 1, 67-100. MR 978378

22. Elias M. Stein, Singular integrals and differentiability properties of functions, Princeton Mathematical Series, No. 30, Princeton University Press, Princeton, N.J., 1970. MR 0290095

23. Hassler Whitney, Analytic extensions of differentiable functions defined in closed sets, Trans. Amer. Math. Soc. 36 (1934), no. 1, 63-89. MR 1501735

Department of Mathematics and Statistics, P.O. Box 35 (MaD), FI-40014 University of JYVÄSKYLÄ, FinLAND

E-mail address: debanjan.s.nandi@jyu.fi

E-mail address: tapio.m.rajala@jyu.fi

E-mail address: timo.m.schultz@student.jyu.fi 